\title{
Use of molecular tools for the identification of males of some scale insects (Hemiptera: Coccoidea), in pheromone traps used for monitoring and comparison with females
}

\begin{abstract}
Species from Pseudococcidae family were studied. It was determined that the dry males of Planococcus citri, and Pseudococcus comstocki, collected by pheromone traps could be useful for the molecular analyses too. The ITS-2 sequences of males and females in case of Pl.citri, Planococcus ficus and Ps. comstocki were identical. This molecular method could differentiate the two mealybug species and this method can be useful to have idea specimens collected by pheromone traps.

Riassunto - Uso degli strumenti molecolari per l'identificazione di maschi di cocciniglie (Hemiptera: Coccoidea), in trappole a feromoni utilizzate per monitoraraggio e comparazione con le femmine.

Sono state prese in considerazione specie appartenenti alla famiglia degli Pseudococcini. E' stato visto che maschi essiccati di Planococcus citri, e Pseudococcus comstocki, raccolti da trappole a feromoni possono essere utilizzati anche per analisi molecolari. La sequenza ITS-2 di maschi e femmine di P.citri, Planococcus ficus e $P$. comstocki è identica. Questo metodo molecolare può differenziare le due specie di cocciniglie e può essere utile per avere un'idea delle specie raccolte in trappole a feromoni.
\end{abstract}

Key words: Planococcus citri, Planococcus ficus, Pseudococcus comstocki, male, pheromone trapping, climate change.

The climate change already supported spread of new pest species, among them a lot of scale insect species (Pseudococcus comstocki (Kuwana), Pseudococcus viburni (Signoret), Planococcus citri (Risso), Planococcus ficus (Signoret), etc, which are very important virus vectors in different crops. These species shows a substantial northward spread in different parts of Europe especially in the last forty years (Ben-Dov et al., 2009; Kozár, 1997, 1998, 2005; Kozár and Nagy Dávid, 1986; Kozár and Szentkirályi, 2005; Pellizzari, 1991; Godinho and Franco, 2001; Sentenac and Kuntzmann, 2003; Boudon-Padieu and Maixner, 2007; Sforza et al, 2005; etc).

The pheromone traps are useful and easy method to study on spread of these pests, although they collect only males. The use of pheromone traps to monitor scale insect 
populations requires some basic knowledge about morphology of males, but the identification of scale insect males is a current problem because all descriptions and keys are based only on females (Kosztarab and Kozár, 1988). Kozár et al. (1996, 1997) prepared morphological and biological keys for males collected by pheromone traps of Diaspidiotus (Quadraspidiotus) perniciosus (Comstock) because it was found that this pheromone compounds are not species specific. This result was verified by molecular method by Frey and Frey (1995). From this point of view the identification problems of the males of Pl. citri, Pl.ficus, and Ps. comstocki taxa collected by pheromone traps could be solved by using more detailed morphological analyses combined with molecular approaches. The aim of this work was (1) to present results of the monitoring of distribution three mealybug species in different parts of Europe, (2) to study the molecular differences of scale insect males of two mealybug species collected by pheromone traps which can occur together easily in the same pheromone traps, (3) to compare the sequences of females and males in case of Pl. citri, Pl.ficus, and Ps. comstocki.

\section{MATERIAL AND METHODS}

Monitoring of mealybugs was conducted by Nagykovácsi type tent trap $(10 \times 10 \mathrm{~cm})$, by using (pheromone ingredients for Pl. citri; (+)-2,2-dimethyl-3-(1-methylethenyl) cyclobutanemethanol acetate, for $P l$. ficus; (S)-lavandulyl senecioate-(S)-lavandulyl isovalerate), for Ps. comstocki; (2,6-dimethyl-1,5-heptadien-3-ol acetate) with Soveurode /Witasek Pflanzenschutz GmbH, Austria/ glue and Biochemtech /Biochemtech Ltd. Kishinev, Moldavia/ pheromone dispensers.

The traps were used in Austria (Wien), Slovakia (Bratislava), Serbia (Belgrade), Greece (Athens, Iraklion, and Chania), Macedonia, and in Hungary in 2009 and 2010, in different times and places (Tab 1).

Tab 1 - Number of mealybug males collected by pheromone traps in 2009*.

\begin{tabular}{|l|c|c|c|c|}
\hline Country, Locality & Time period & $\begin{array}{c}\text { Planococcus } \\
\text { citri }\end{array}$ & $\begin{array}{c}\text { Planococcus } \\
\text { ficus }\end{array}$ & $\begin{array}{c}\text { Pseudococcus } \\
\text { comstocki }\end{array}$ \\
\hline $\begin{array}{l}\text { Hungary, Budapest, } \\
\text { Ördögárok ú. }\end{array}$ & $04.28-07.31 .2009$ & 0 & 0 & 0 \\
\hline $\begin{array}{l}\text { Hungary, Budapest, } \\
\text { Ördögárok ú. }\end{array}$ & $07.31-09.02 .2009$ & 8 & 2 & 1 \\
\hline $\begin{array}{l}\text { Hungary, Budapest, } \\
\text { Ördögárok ú. }\end{array}$ & $09.02-10.02 .2009$ & 0 & - & 0 \\
\hline $\begin{array}{l}\text { Hungary, Budapest, } \\
\text { Ördögárok ú. }\end{array}$ & $10.02-11.02 .2009$ & 3 & - & 2 \\
\hline $\begin{array}{l}\text { Hungary, Budapest, } \\
\text { M0,Csepel }\end{array}$ & $05.18-03.08 .2009$ & 0 & 0 & 0 \\
\hline $\begin{array}{l}\text { Hungary, Budapest, } \\
\text { M0,Csepel }\end{array}$ & $03.08-03.09 .2009$ & 2 & 0 & 0 \\
\hline $\begin{array}{l}\text { Hungary, Budapest, } \\
\text { M3, Szilas }\end{array}$ & $05.11-07.30 .2009$ & 0 & 0 & 0 \\
\hline
\end{tabular}




\begin{tabular}{|c|c|c|c|c|}
\hline $\begin{array}{l}\text { Hungary, Budapest, } \\
\text { M3, Szilas }\end{array}$ & 05.11-07.30.2009 & 0 & 0 & 0 \\
\hline $\begin{array}{l}\text { Hungary, Budapest, } \\
\text { M3, Ecséd }\end{array}$ & 05.11-07.30.2009 & 0 & 0 & 0 \\
\hline $\begin{array}{l}\text { Hungary, Budapest, } \\
\text { M3, Ecséd }\end{array}$ & 07.30-09.09.2009 & 1 & 0 & 0 \\
\hline $\begin{array}{l}\text { Hungary, Budapest, } \\
\text { M5, Kecskemét }\end{array}$ & 05.14-07.28.2009 & 0 & 0 & 0 \\
\hline $\begin{array}{l}\text { Hungary, Budapest, } \\
\text { M5, Kecskemét }\end{array}$ & 07.28-09.03.2009 & 0 & 0 & 0 \\
\hline $\begin{array}{l}\text { Hungary, Budapest, } \\
\text { M5, Röszke }\end{array}$ & 05.14-07.28.2009 & 0 & 0 & 0 \\
\hline $\begin{array}{l}\text { Hungary, Budapest, } \\
\text { M5, Röszke }\end{array}$ & 07.28-09.03.2009 & 0 & 0 & 0 \\
\hline $\begin{array}{l}\text { Hungary, Budapest, } \\
\text { M7, Budaörs }\end{array}$ & 05.06-07.30.2009 & 0 & 0 & 0 \\
\hline $\begin{array}{l}\text { Hungary, Budapest, } \\
\text { M7, Budaörs }\end{array}$ & 07.28-08.29.2009 & 1 & 0 & 0 \\
\hline $\begin{array}{l}\text { Hungary, Budapest, } \\
\text { M7, Velence }\end{array}$ & 05.06-07.24.2009 & 0 & 0 & 0 \\
\hline $\begin{array}{l}\text { Hungary, Budapest, } \\
\text { M7, Velence }\end{array}$ & 07.08-08.31.2009 & 2 & 1 & 1 \\
\hline $\begin{array}{l}\text { Hungary, Budapest, } \\
\text { M7, Töreki }\end{array}$ & 05.06-07.25.2009 & 0 & 0 & 0 \\
\hline $\begin{array}{l}\text { Hungary, Budapest, } \\
\text { M7, Töreki }\end{array}$ & 07.25-10.09.2009 & 3 & 1 & 2 \\
\hline $\begin{array}{l}\text { Hungary, Budapest, } \\
\text { M7, Letenye }\end{array}$ & 05.06-07.25.2009 & 0 & 0 & 0 \\
\hline $\begin{array}{l}\text { Hungary, Budapest, } \\
\text { M7, Letenye }\end{array}$ & 07.25-10.09.2009 & 0 & - & 0 \\
\hline $\begin{array}{l}\text { Hungary, Nagykovácsi, } \\
\text { around greenhouse }\end{array}$ & 04.28-07.31.2009 & 22 & 0 & 0 \\
\hline $\begin{array}{l}\text { Hungary, Nagykovácsi, } \\
\text { around greenhouse }\end{array}$ & 07.31-09.06.2009 & 95 & 1 & 2 \\
\hline $\begin{array}{l}\text { Hungary, Nagykovácsi, } \\
\text { in greenhouse }\end{array}$ & 05.10-06.10.2009 & 8 & 0 & 0 \\
\hline Austria, Wien & 04.03-07.29.2009 & 0 & 0 & 0 \\
\hline Austria, Wien & 07.29-08.28.2009 & 3 & 0 & 0 \\
\hline $\begin{array}{l}\text { Greece }^{\mathrm{x}} \text {, } \\
\text { Olympus Plaza }\end{array}$ & 09.20-09.26.2009 & 0 & - & 0 \\
\hline Greece, Kifissia & 09.20-09.26.2009 & 14 & - & 0 \\
\hline Greece, Iraklion & 09.20-09.25.2009 & 48 & - & $41 !$ \\
\hline $\begin{array}{l}\text { Makedonia, } \\
\text { Vardar bridge }\end{array}$ & 09.20-09.27.2009 & 0 & - & 0 \\
\hline Slovakia, Bratislava & 07.29-08.28.2009 & 102 & 0 & 0 \\
\hline Switzerland, Lausane & 06.16-06.28.2009 & 0 & 0 & 0 \\
\hline
\end{tabular}

* Between 04.04-04.10, 2010 the traps in Serbia (Beograd), Greece (Gevgelija, Thessaloniki, Olympus plaza, Kifissia, Chania), and in Hungary (Budapest) between 05.01-05.31, 2010, did not collected males of these three species. 


\section{DNA extraction, amplification, cloning and sequencing}

Total genomic DNA was extracted from single individuals (males and females) using REDExtract-N-Ampl ${ }^{\mathrm{TM}}$ Tissue PCR Kit (Sigma) according to manufacturer instruction. Females and males of $P$. citri, $P$. ficus and $P$. comstocki were tested (Tab 2.). After preliminary experiments CAS5p8sFc (sense) (5'-gcgaacatcgacaagtcgaacgcacat-3') and CAS28sB1d (antisense) (5'-ttgttttcctccgcttattaatatgcttaa-3') primer pair (Kim and Lee 2008) was selected for PCR. The primers amplified the ITS 2 sequence of nuclear DNA. PCR was performed using Taq DNA polymerase (Fermentas) in thermo-cycler (Eppendorf Mastercycler gradient) according to the following procedure: initial denaturation at $95^{\circ} \mathrm{C}$ for $4 \mathrm{~min}$, followed by 40 cycles of $95{ }^{\circ} \mathrm{C}$ for $30 \mathrm{sec}$, annealing temperature $50{ }^{\circ} \mathrm{C}$ for $30 \mathrm{sec}$, extension at $72{ }^{\circ} \mathrm{C}$ for $60 \mathrm{sec}$; final extension at $72{ }^{\circ} \mathrm{C}$ for $10 \mathrm{~min}$. The PCR products were purified using Gel/PCR DNA Fragments Extraction Kit (Geneaid). Purified PCR products from 3 dry individuals of $P$. citri from pheromone traps, and three females living on Ficus benjamina (Budapest) and 3 females of $P$. comstocki from mass culture on potato (Van), and three males from ethanol from mass culture on potato (Van). Pl.ficus individuals ( 2 females and 2 males) were from mass culture on potato (Van). Each were cloned into CloneJet (Fermentas) vector and inserted into Escherichia coli DH5a competent cells. All cloning steps were based upon standard molecular biology protocols (Sambrook et al. 1989). The recombinant plasmids isolated from selected colonies were sequenced using pJET1.2 forward and reverse primers, the PCR products of two P. citri, and P. comstocki were sequenced by CAS5p8sFc and CAS28sB1d primers by an automated DNA sequencer (Applied Biosystem Gene Analyzer 3100). Sequence comparisons were performed using Wisconsin Package version 10.0 Genetic Computer Group (GCG) sequence analysis software (Devereux et al. 1984).

\section{RESULTS AND DISCUSSION}

The pheromone trap catches show that only Pl. citri males were collected (Tab 1.) and this species was caught only in Greece (Kifissia, Iraklion) in field conditions. In other places such as in Hungary (Nagykovácsi), or Slovakia (Bratislava) the insects caught on outdoor plants probable originate from greenhouses plants. In these places, this species probably could not overwinter, or may be only in mild winter.

The males collected by Ps. comstocki pheromone traps in Greece (Crete, Iraklion) belongs to $P l$. citri according to the molecular data, which was abundant in park on Catalpa sp., Ficus sp., Morus sp. and other plants, and their males could appear accidentally in Ps. comstocki traps.

On the other hand Pl.ficus and Ps. comstocki were not found in studied places and times. The one or two males caught by traps in some places probable belong to other genera and species. 


\begin{tabular}{|c|c|c|c|c|c|c|c|c|c|c|c|c|c|c|c|c|c|c|c|c|c|c|}
\hline 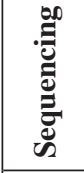 & $\begin{array}{l}\frac{0}{0} \\
\bar{\infty}\end{array}$ & $\begin{array}{l}8 \\
0 \\
0 \\
\infty\end{array}$ & $\begin{array}{l}8 \\
\overrightarrow{0} \\
0\end{array}$ & $\begin{array}{l}\text { हे } \\
\vec{\infty}\end{array}$ & $\begin{array}{l}0 \\
0 \\
n \\
n\end{array}$ & $\begin{array}{l}\text { مे } \\
\text { के }\end{array}$ & $\begin{array}{l}\text { مे } \\
\text { के }\end{array}$ & $\begin{array}{l}\text { ڤे } \\
\text { مे}\end{array}$ & 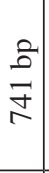 & $\begin{array}{l}2 \\
\stackrel{2}{2}\end{array}$ & $\begin{array}{l}\text { 今े } \\
\stackrel{2}{N}\end{array}$ & $\begin{array}{l}\text { वे } \\
\frac{\Delta}{7}\end{array}$ & $\begin{array}{l}\frac{0}{2} \\
\stackrel{2}{r}\end{array}$ & 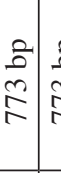 & 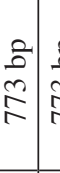 & 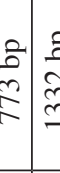 & 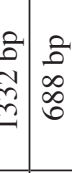 & $\left|\begin{array}{l}0 \\
\infty \\
\infty \\
0 \\
0\end{array}\right|$ & $\begin{array}{l}2 \\
0 \\
0 \\
0 \\
0\end{array}$ & $\begin{array}{l}\text { مे } \\
\text { aे }\end{array}$ & \begin{tabular}{l}
2 \\
\multirow{2}{2}{} \\
$\stackrel{2}{2}$
\end{tabular} & \\
\hline 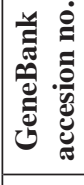 & $\begin{array}{l}\infty \\
0 \\
\infty \\
0 \\
\text { ஸे } \\
\vdots \\
\Xi \\
\end{array}$ & 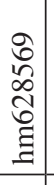 & $\begin{array}{l}尺 \\
n \\
\infty \\
i \\
0 \\
\vdots \\
\vdots\end{array}$ & $\begin{array}{l}\vec{n} \\
\infty \\
\tilde{b} \\
\vdots \\
\vdots\end{array}$ & 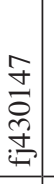 & 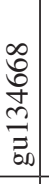 & 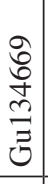 & & & 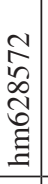 & & & 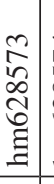 & 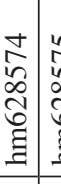 & 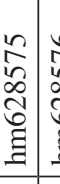 & 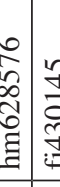 & 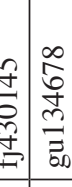 & 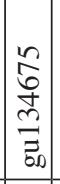 & 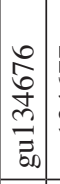 & 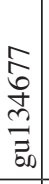 & & \\
\hline 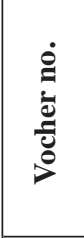 & 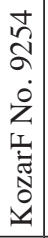 & 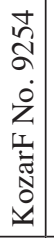 & 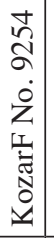 & 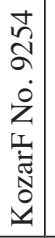 & & $\begin{array}{l}1 \\
8 \\
8 \\
8 \\
8 \\
8 \\
8\end{array}$ & & 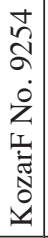 & 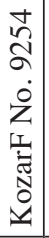 & 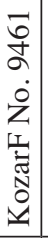 & 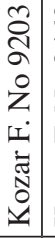 & 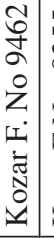 & 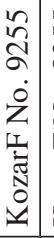 & 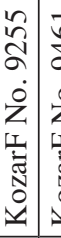 & 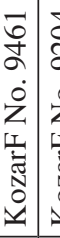 & 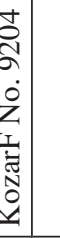 & 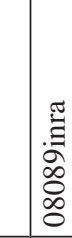 & 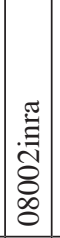 & 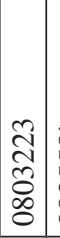 & 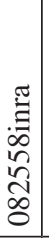 & 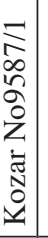 & 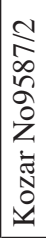 \\
\hline$\stackrel{\circ}{2}$ & 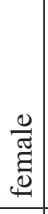 & 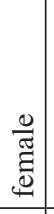 & $\begin{array}{l}\tilde{E} \\
\dot{0} \\
\underline{\tilde{U}} \\
\underline{\Xi}\end{array}$ & 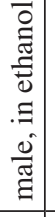 & & & & 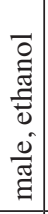 & 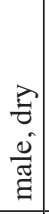 & 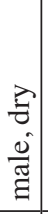 & 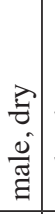 & 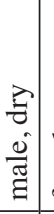 & 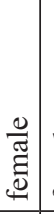 & 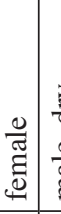 & 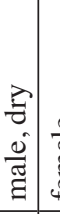 & 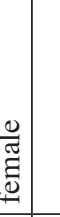 & & & & & $\begin{array}{l}\frac{0}{\tilde{J}} \\
\stackrel{\Xi}{\Xi}\end{array}$ & 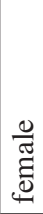 \\
\hline ס & 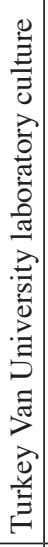 & 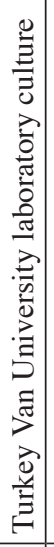 & 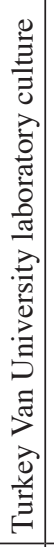 & 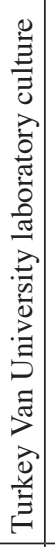 & 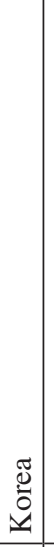 & 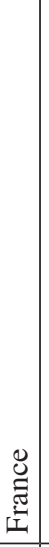 & 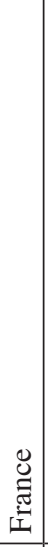 & 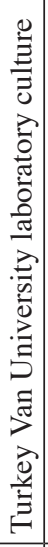 & 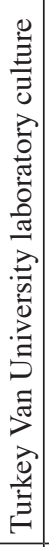 & 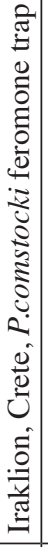 & 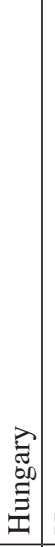 & 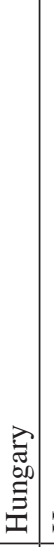 & 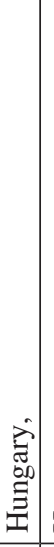 & : & 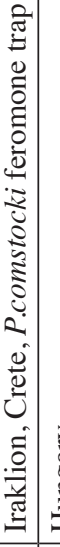 & 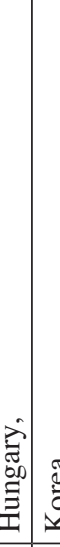 & 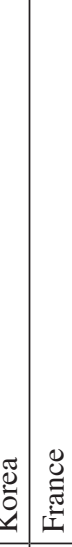 & 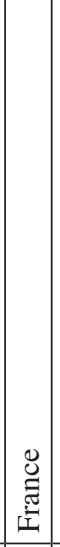 & 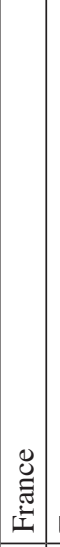 & 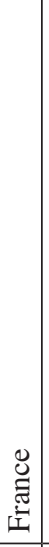 & 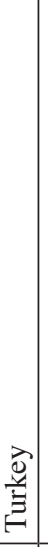 & $\begin{array}{l}\text { 仓े } \\
\text { 音 } \\
\text { E }\end{array}$ \\
\hline 岕 & 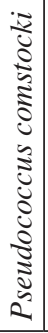 & 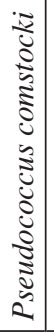 & 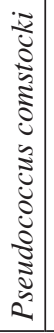 & 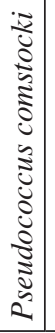 & 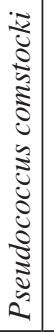 & 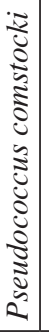 & 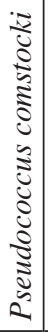 & $\begin{array}{c}\vec{a} \\
0 \\
0 \\
\vdots \\
\vdots \\
0 \\
0 \\
0\end{array}$ & $\begin{array}{l}0 \\
0 \\
0 \\
\vdots \\
\vdots \\
0 \\
0 \\
\dot{0} \\
2\end{array}$ & 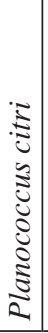 & 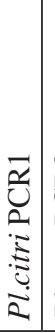 & 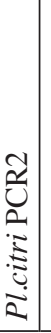 & 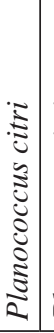 & 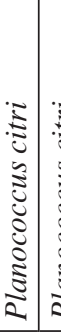 & 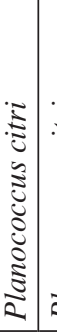 & 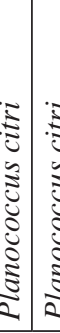 & 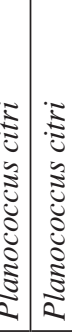 & 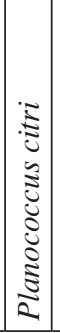 & 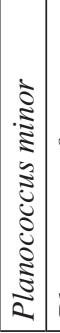 & 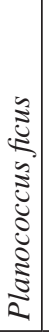 & 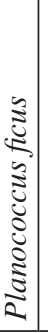 & 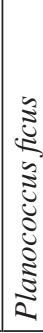 \\
\hline
\end{tabular}




\section{Characteristics of sequences}

It was determined that the used DNA extraction procedure was suitable to handle adequate quantity and quality DNA from dry males from pheromone traps (6-7 months kept at laboratory condition), and males from ethanol or from living males and females.

The PCR product size (without the primers) of ITS2 sequences were $801 \mathrm{bp}$ for $P$ s. comstocki, 773 bp for $\mathrm{Pl}$. citri and $770 \mathrm{bp}$ for Pl.ficus. The sequence variation among Ps. comstocki males and females were between $0.375-0.0$ and among $P l$. citri between 0.26 and 0.00 (See Tab 3. and 4.). The amplified ITS2 sequences were compared with the similar sequences in the Genebank. It is very interesting that Ps.comstocki collected and characterized in Korea (1357 bp Genebank accession no. fj430147), and France (709 bp Genebank accession no.gu134668 and gu134669) showed very high identity with species collected in Turkey (in case of gu134668 and hm628568 there is $100 \%$ ) The same tendency could be observed in the case of Pl. citri and Pl. ficus. Pl. citri individuals collected and characterized in France, Korea or Hungary showed 99.9 - $100 \%$ identity. ITS2 sequences of $P l$. ficus individuals also showed $99.9-100 \%$ identity regardless of their origin, but differed from Pl. citri (90.4\% identity). Pl. minor were also included in the comparison test and showed $99.7 \%$ identity with $P$ l. citri. Considerable divergence was observed between the Ps. comstocki and Pl. citri (53.3\%-56.1\% identity), which give possibility to identify and distinguish the males of these species (Fig. 1).

The very low sequence variation of different individuals regardless their origin and state (living, dry material or conserved in ethanol) indicate that ITS-2 molecular marker is suitable method for reliable characterization and differentiation of Pl. citri and Ps. comstocki species. It was determined that males from the pheromone traps kept in 6-7 months in laboratory condition were suitable for molecular work. On basis of molecular data it was possible to separate the males of Ps. comstocki and Pl.citri. The high divergence for the ITS 2 region suggest that it should be possible to design species-specific primers and discriminate Ps. comstocki and Pl. citri males based on PCR method.

The molecular data could be a very important tool for the verification of the males collected by pheromone traps, to exclude the accidentally present specimens in the trap, and other species, for which the pheromone compound could serve as attractant. This result is especially important in quarantine and ecological studies of distribution of important pest species in new localities.

\section{ACKNOWLEDGEMENTS}

The authors are grateful to the Hungarian Fund for Research (OTKA) (No. 75889) for financial support for this work, as well as to Dr. Panos Milonas and Zsuzsanna Konczné Benedicty for help in trapping works in Greece and in Hungary. 


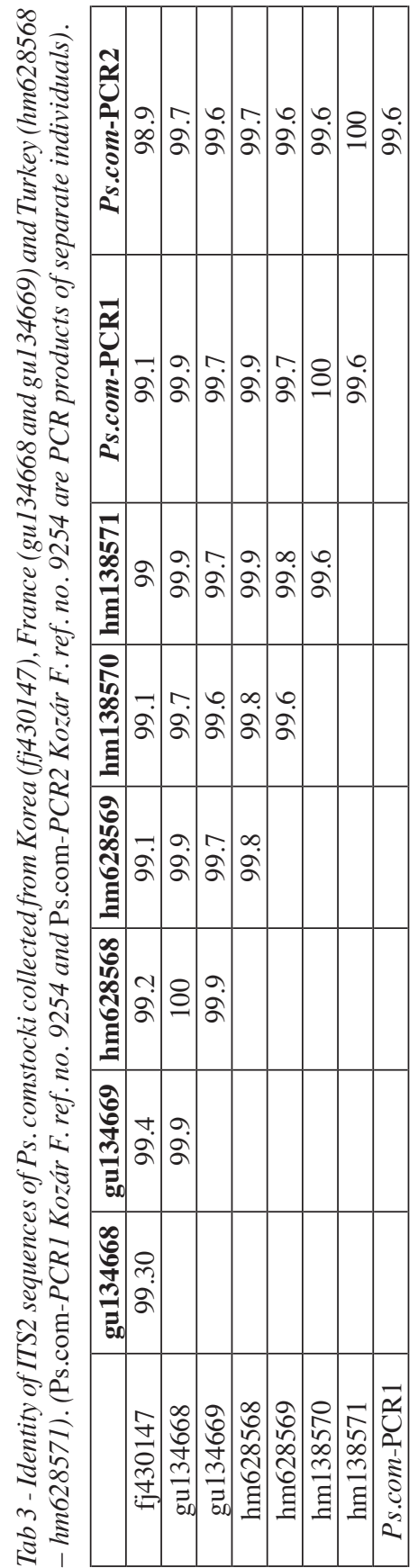

\begin{tabular}{|c|c|c|c|c|c|c|c|c|c|c|c|c|c|}
\hline$\sum$ 文 & $\begin{array}{l}0 \\
n \\
n \\
n\end{array}$ & $\begin{array}{l}m \\
\cdots \\
n\end{array}$ & $\begin{array}{l}n \\
n \\
n \\
n\end{array}$ & $\begin{array}{ll}0 \\
\bullet \\
\bullet\end{array}$ & $\begin{array}{lll}a & 5 \\
n & n & \end{array}$ & \begin{tabular}{l|l}
0 \\
0 \\
0 \\
$n$
\end{tabular} & & $\begin{array}{ll}-1 \\
\bullet \\
\bullet\end{array}$ & $\begin{array}{l}a \\
g \\
\end{array}$ & 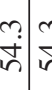 & \begin{tabular}{l|l}
$f$ \\
\hdashline \\
\hdashline
\end{tabular} & in & - \\
\hline 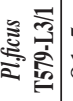 & na & $\begin{array}{l}0 \\
8 \\
8\end{array}$ & $\begin{array}{l}+ \\
\dot{\leftrightarrow}\end{array}$ & 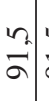 & 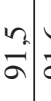 & \begin{tabular}{l|l}
0 & $=$ \\
$\bar{a}$ &
\end{tabular} & ą) & & ฉి & $8 \mid \stackrel{\circ}{8}$ & ho & & \\
\hline 憂 & $\begin{array}{l}+ \\
\dot{a}\end{array}$ & $\begin{array}{l}+ \\
8 \\
8\end{array}$ & ?.: & $\begin{array}{l}\sigma_{0} \\
\vec{a}\end{array}$ & $\begin{array}{c}m \\
\bar{a}\end{array}$ & $\frac{n}{2}$ & 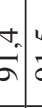 & कृ: & $\begin{array}{lll}+ \\
8\end{array}$ & बे & $=\infty$ & & \\
\hline 怤 : & ¿ & 8 & बे. & 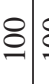 & ¿ & ¿ & 8 & 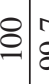 & $\hat{\sigma}$ & $\begin{array}{c}\dot{t}_{0} \\
\dot{8}\end{array}$ & & & \\
\hline 롤 & 8 & 8 & बे. & 8 & 8 & 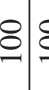 & 8 & 8 & बे & 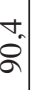 & & & \\
\hline$E_{0} \underset{0}{0}$ & $\begin{array}{l}+ \\
\dot{\Omega} \\
\varnothing\end{array}$ & $\begin{array}{l}+ \\
\dot{8}\end{array}$ & ๙ุ. & $\begin{array}{l}+ \\
\dot{\Omega}\end{array}$ & $\begin{array}{l}\sigma_{+} \\
8\end{array}$ & $\begin{array}{l}\sigma_{f} \\
\delta\end{array}$ & \&. & $\begin{array}{l}+ \\
\delta \\
\delta\end{array}$ & $\begin{array}{l}\nabla_{0} \\
8\end{array}$ & & & & \\
\hline 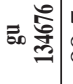 & $\hat{\delta}$ & 8 & $\begin{array}{l}0 \\
\text { aे }\end{array}$ & $\hat{\sigma}$ & $\hat{2}$ & s. & & & & & & & \\
\hline 玉 福 & 8 & 8 & बे. & $\hat{\sigma} \mid \delta$ & & 8 & ลे. & & & & & & \\
\hline 王点 & $\partial े$ & 8 & बे & $\stackrel{-}{2}$ & $\stackrel{\circ}{\circ}$ & & & & & & & & \\
\hline 至 & 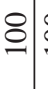 & \& & बे & $\begin{array}{l}\hat{\sigma} \\
\text { aे }\end{array}$ & 2 & & & & & & & & \\
\hline 玉 & รे. & 8 & बे & 8 & & & & & & & & & \\
\hline 玉 & aे & 8 & aे & & & & & & & & & & \\
\hline 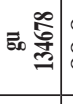 & $\hat{\sigma}$ & & & & & & & & & & & & \\
\hline$\Xi_{0}$ & 8 & & & & & & & & & & & & \\
\hline & 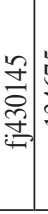 & 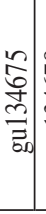 & $\begin{array}{cc}\infty \\
0 \\
6 \\
+ \\
0 \\
\vdots \\
\vdots \\
0.0\end{array}$ & 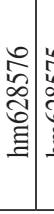 & 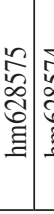 & 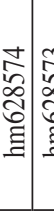 & 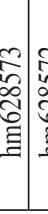 & & 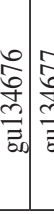 & 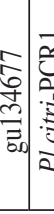 & 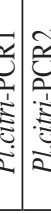 & 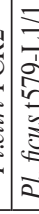 & 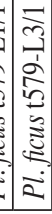 \\
\hline
\end{tabular}


Fig. 1 - Comparison of ITS 2 sequences of Ps. comstocki and Pl. citri males.

Percent Similarity: 55.906 Percent Identity: 55.906

Match display thresholds for the alignment(s):

| = IDENTITY

: $=5$

. $=1$

Pcomstockif.seg x PlanococcuscitriA5.seg January 12, 2011 09:56

1 TGCGGCCCTCGTG. ACCAAAGAGTCCTGGGCCACGCCTGTCTGAGGGTCG 49 ||||||||||| | || ||||||||||||||||||||||||||||||||||||

1 TGCGGCCCTCGCGAaCAAAAGAGTCCTGGGCCACGCCTGTCTGAGGGTCG 50

50 GTCATACGTGTAACACGATGGTTGCGTTCTCGCGAGTTTCGCGCTCGCTC 99 || ||||||||||||||||||||||||||||||||| | ||| |

51 GTTATACGTGTAACACGATGGTTGCGTTCTCGCGAGCGCCTCGCAGTTTT 100

100 CGCAGCAGACCAGATCGTGCGCGCTCGTCGCGCGCGTGTGTGtgAATTCA 149

101 CACCGCGAGGTTAGCTCGCCCCGTGACAGACCAGATCGAGCGTGTGTTTA 150

150 CGCACGCCGCCGAG. . . CGCGCGAGAGCGAGTACGTTGCGCTTTGCGGCG 196 |||| | || || || || || | || |

151 CGCACATGCCAGAGCGACGCGGTTTTCGAATCGCGTCGCTCGAAGCTGAC 200

197 ACTTCGTACGCTCGAAGCTGACGATTCGTTTGCCCGCGTGACTTGTCGCG 246

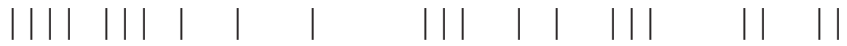

201 GACTCGTTCGCGCTGACGCGGGCCATCGCCTCCGTGCGGTGGTTAGCGTC 250

247 TCGACGGCGAACGTTCGTCGAAATATTGCGGTTCTCGCCCGCATCGATAG 296

| || ||||||||||||| |||||| | | ||| ||

251 GTG.CGTCGAACGTTCGTTGAAATACACGGCGCCGATGCCGAGAAACAAG 299

297 TTCGTTCACGAACAACAACGTGTTCGACGAAAGCGTGCCGCGTGCAGCGG 346 |||||||||||||| | || || | ||| |

300 TTCGTTCACGAACACCGTGTTCGAAAACGTTGCCGCGTATAGTGGTAGAG 349

347 ACCATATGCGAACGCGATGCGGTAGATACTCGGAGAGTGCGTTGGCCGTC 396 | | | || || || || || || | ||

350 AgCGAAGGCGGTAGCTATCGTAGAGAGCGTCCGATGAACGGGCATCCAGA 399

397 GGATGTACGGGCGTCGAAAATTCTCTCTCGGGGCGACGGCGGGTGACGCG 446 | | | || || | | | |||

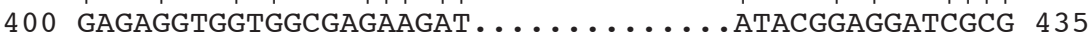


447 CGTGTTGTACGATCGCGCGTTCC........AACACCCCCTCGTCGCTTGA 489<smiles>CCCCCCCCCCCCCC</smiles>

436 CGTGTTGTACGATCGCGCGTCTCCGAAAAAAAAAGCTTAACGTTACCGCC 485

490 GAAAAAAATTATACGACGCGAAGACGGTTTTCTCTTTACACGCCAACCGC 539

486 GTCGAATCTCATACGATGCGAAGACGTCTCTCGAAATAGAGGCGATAGTT 535

540 GATCGTTTCCCGATCGGACTTTGAGTATTTTTTCCGCAGTTCGCCCGCCT 589

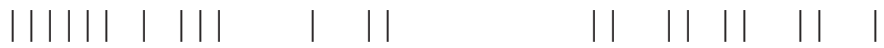

536 TGCCGTTTCTCTATCCACGTACGA.......... CGTTGTACGTGCGATT 575

590 GCACGACGACGTTGGCGCACGTACGCGCGCGCGTTAGCGTGTGTTCGCGT 639 || || |||| ||| || | || | | |||||

576 GCGCG...... CGTTCGCGTCGTTATTCCGGCCGTTGTCGACGAATTCGCTC 621

640 CGTCATTCCGGCCGTTGTCGACGAAATTCAATAATTCGAAGACGGCACTC 689

622 ACGCGTCATGTACGGACGCGATGATGACGATCGAGAACGGGCACACATTC 671

690 GGAAGGCGATTTCGCGACTCGCGTTACGTGCGACGCACGCGAACGTACCC 739

672 GGAAGACGA.TGCGAGCGTGCGATAATTCGCGTTATAAGCGTACTT. . . 716

740 GACACACACGCACGCATACGATTTCACGCCGACCTCAGATCAGGTAAGAC 789

717 ... CGTACCAGGCTTCACTCTGTATCACGCCGACCTCAGATCAGGtAAGAC 764

790 TACCCGCCGAAT 801

| || || || |

765 TACCCGCCG... 773 


\section{REFERENCES}

Ben-Dov Y., Miller D. R., Gibson G. A. P., 2009 - ScaleNet: a database of the scale insects of the World. Source: http//www.sel.barc.usda.gov/scalenet/scalenet.htm, using - Scales in a Region Query Results.

Beuning L., Murphy P., Wu E., Batchelor T., Morris B., 1999 - Molecular-based approach to the differentiation of mealybug (Hemiptera: Pseudococcidae) species. -Journal of Economic Entomology, 92, 463-472.

Devereux J., Haeberli P., SMithis O., 1984 - A comprehensive set of sequence analysis program for the VAX. - Nucleic Acids. Res., 12, 287-395.

FREY J., FREY B., 1995 - Molecular identification of six species of scale insects (Quadraspidiotus sp.) by RAPD-PCR: assessing the field-specificity of pheromone traps. - Molecular Ecology, 4, 777-780.

Godinho M. A., Franco J.C. 2001- Survey of the pest status of mealybugs in Portugese vineyards. IOBC WPRS Bulletin, 24, 221-226.

KIM H., LEE S., 2008 - Molecular systematics of the genus Megoura (Hemiptera: Aphididae) using mitochondrial and nuclear DNA sequences. - Molecules and Cells, 25: 510-522.

Kosztarab M., KozÁr F., 1988 - Scale insects of Central Europe - Akadémiai Kiadó, Budapest, $456 \mathrm{pp}$.

KozÁR F., 1997 - Insects in a Changing World. - Acta Phytopathologica et Entomologica Hungarica, 32, 129-139.

KozÁr F., (ed.) 1998 - Catalogue of Palaearctic Coccoidea. - Plant Protection Institute, Hungarian Academy of Sciences, Budapest, 526 pp.

KozÁr F., 2005 - Distribution records of scale insects in Hungary. - MTA Növényvédelmi Kutatóintézete, Budapest, 136 pp.

KozÁR F., HipPE C., MANI E., 1995 - Quadraspidiotus nembe tartozó pajzstetú fajok hímjeinek morfológiai vizsgálata (Homoptera: Coccoidea) (in Hungarian) (Morphological study of males of the Quadraspidiotus genus). In: Sáringer Gy., Seprôs I. és Szemessy Á. (szerk.). 41. Növényvédelmi Tudományos Napok, Budapest. 51.

KozÁr F., HiPPE C., MANI E., 1996 - Morphometric analyses of the males of Quadraspidiotus species (Hom., Diaspididae) found in European orchards or their vicinity. - Journal of Applied Entomology, 120, 433-437.

KoZÁR F., HipPe C., MANI E., 1997 - Kulcs a Quadraspidiotus (Homoptera: Coccoidea) pajzstetű nembe tartozó hímek meghatározásához (in Hungarian with English summary) (Key to determine Quadraspidiotus (Homoptera: Coccoidea) males and to distinguish them from males of other genera). - Növényvédelem, 33, 321-327.

KoZÁR F., NAGY DÁVID A., 1986 - The unexpected northward migration of some species of insectes in Central Europe and the climatic changes. - Anzeiger für Schadlingskunde, Planzenschutz und Umweltschutz, 59, 90-94.

KOZÁR F., SZENTKIRÁLYI F., 2005 - Some effects of climate change on Insects in Hungary. In: Láng I. (ed.) - Natural ecosystems, CD, 208-218.

Lazar J., Kölber M., Farkas E., Farkas G., Tobias L., 2000 - Grapevine virus diseases and clean grape stock program in Hungary. - 13th ICVG Conference, Adelaide, 12-17 March 2000.

Malausa T., Fenis A., Warot S., Germain J.F., Ris. N., Prado E., Botton M., VanlerbergheMasutti F., Sforza R., Cruaud C., Couloux A., Kreiter, P., 2010 - DNA markers to disentangle complexes of cryptic taxa in mealybugs (Hemiptera: Pseudococcidae) - Journal of Applied Entomology, 135(1-2):142-155. 
Park D-S., LeEm Y.J., Hahn K.W., SuH S-J., Hong K-J., OH H-W., 2010 - Molecular identification of mealybugs (Hemiptera: Pseudococcidae) found on Korean Pears. - Journal of Economic Entomology, 103, 25-33.

PellizZARI Scaltriti G., 1991 - Recent data on Italian fauna of Homoptera Coccoidea. Atti XVI Congresso nazionale italiano di Entomologia, Bari - Martina Franca (Ta) 23/28 settembre 1991, 763-769.

Rung A., Scheffer S. J., Evans G., Miller D., 2008 - Molecular identification of two closely related species of mealybugs of the genus Planococcus (Homoptera: Pseudococcidae). Annals of the Entomological Society of America, 101, 525-532.

SAMBrook J., Fritsch E.F., MANiATIS T.A., 1989 - Molecular cloning: a laboratory manual. - Cold Spring Harbor Laboratory Press, Cold Spring Harbor, NY, 1659 pp.

Sentenac G., Kuntzmann P., 2003 - Etude des Cochenilles et des antagonistes qui leur sont associés dans des vignobles en Bourgogne et en Alsace de 2000 à 2002. - IOBC WPRS Bulletin, 26, 247-252.

SForza R., Boudon-PAdieu E., GReIF C. 2003 - New mealybug species vectoring grapevine leafroll associated viruses 1 and 3 (GLRaV-1 and 3). - European Journal of Plant Pathology, 109, 975-981.

IstVÁn TóBIÁs - Plant Protection Institute, Hungarian Academy of Sciences, H-1525, P.O.Box 102, Budapest, Hungary. tobias@julia-nki.hu

FERENC KozÁR - Plant Protection Institute, Hungarian Academy of Sciences, H-1525, P.O.Box 102, Budapest, Hungary.

BoRa. M. KAYDAN - Yüzüncü Yıl Üniversity, Agriculture Faculty, Plant Protection Department, 65080 Campus, VAN.

KInga FetyKó - Plant Protection Institute, Hungarian Academy of Sciences, H-1525, P.O.Box 102, Budapest, Hungary.

Accepted 20 December 2010 